\title{
Investigation of the chemical structure of our Galaxy using radial pulsating stars as tracers
}

\author{
Marian Doru Suran \\ Astronomical Institute of the Romanian Academy, str. Cutitul de Argint 5, RO-040557, \\ Bucharest, Romania \\ email: suran@aira.astro.ro
}

\begin{abstract}
In this paper we try to determine the properties of different galactic components $(2<r<12 k p c)$ using radial pulsating stars (RPS stars) as tracers. For RPS stars in Galaxy we use a quasi-seismological inversion method (Post Theoretical Mass Method, PTM) which allows us to determine the mass $M$ and the abundance $Z$ for each star. Based on PTM method and using RPS stars as tracers we could determine the chemical structure and evolution of the Galaxy: abundance gradients and segregation between different evolutionary populations. This method is planned to be extended further using the RPS star observed by the KEPLER space mission.
\end{abstract}

Keywords. ISM: abundances

\section{Abundance gradients in Galaxy using Cepheids as tracers}

Abundance gradients across different kind of galaxies provide fundamental constraints on the chemical evolution of these objects and on the plausibility of the physical assumptions adopted in chemical evolution models. Although the abundance gradients have been the cross-road of several empirical and theoretical investigations, we still lack quantitative constraints on the observed spread in chemical composition at fixed distances in galaxies. Moreover, we need to assess whether different tracers to study the fine structure of the abundance gradients.

Cepheids, when compared with other tracers in galaxies (open clusters, HII regions, $B$-type stars, PN) present several advantages to evaluate elements abundances in different kind of galaxies:

- They are luminous and easily identified objects;

- They are ubiquitous across galaxies;

- Their spectra show a wealth of well defined lines, therefore, accurate abundance measurements of iron and heavy elements can be provided.

In this paper we use two methods to determine abundances of the Cepheid stars:

- The theoretical inversion (quasi-asteroseismic) method (Post Theoretical Mass Method PTM);

- The observational spectral method.

\section{PTM theoretical method}

The Post-theoretical Mass Method represents a combination of the pulsational and evolutive mass relations and represents an extension of the Theoretical Mass Method of Cox (1980), using the equations:

$$
P_{o b s}=P_{t h}\left[\left(M, L, T_{e}\right),(Y, Z),\left(l_{M L T}, \kappa, E O S\right)\right]
$$




$$
\begin{aligned}
T_{e} & =T_{e}\left[A_{g}\left(a_{g}, b_{g}\right)\right] \\
\log L_{p u l s} & =f_{1}\left[\left(P, T_{e}, M\right),(Y, Z), \Delta\right]=f_{1}\left[\left(P, T_{e}, M\right),\left(Y, a_{Z}, b_{Z}\right), \Delta\right] \\
\log L_{e v} & =f_{2}\left[\left(M, t_{9}\right),(Y, Z),\left(l_{M L T}, \kappa, E O S\right)\right]
\end{aligned}
$$

where $(Y, Z)$ represents the abundances in He and heavy metals $([F e / H] \propto[Z / X])$, t9 age of the star; $\left(l_{M L T}, \kappa, E O S\right)$ - the ingredients of the stellar evolutive calculations; $\left(M, L, T_{e}\right)$ - the physical parameters of the star, and where

- The first relation represents the asteroseismic relation between the observed and theoretical period values (radial pulsating modes), where the theoretical period was inferred from a linear non-adiabatic model (Suran 1985, 1986, 1990).

- The second relation is a temperature-amplitude relation in a $g$ pass-band: $\log T_{e}=$ $a_{g} A_{g}+b_{g}$

- The third relation must be a variant of the general $\left(P-L-M-T_{e}-Y-Z\right)$ relation:

$$
\log L=a_{0} \log M+\left[a+b \log P+c \log T_{e}\right]+d Y+e[F e / H]+f \Delta
$$

- The fourth relation represents the accommodation of our results with the points in the evolutive HR diagram. The input parameters are: $\left\{P, A_{g}\right\}$ and the output parameters are: $\left\{\left(M, L, T_{e}\right),[Z / X]\right\}$.

\section{PTM method results}

PTM method application: 9 Cepheids in Galactic disk $\left(6.5<R_{G}<10.5\right)$ and abundance gradient -0.0203 dex, 7 Cepheids in Galactic disk $\left(6.5<R_{G}<8.5\right)$ and abundance gradient -0.0653 dex.

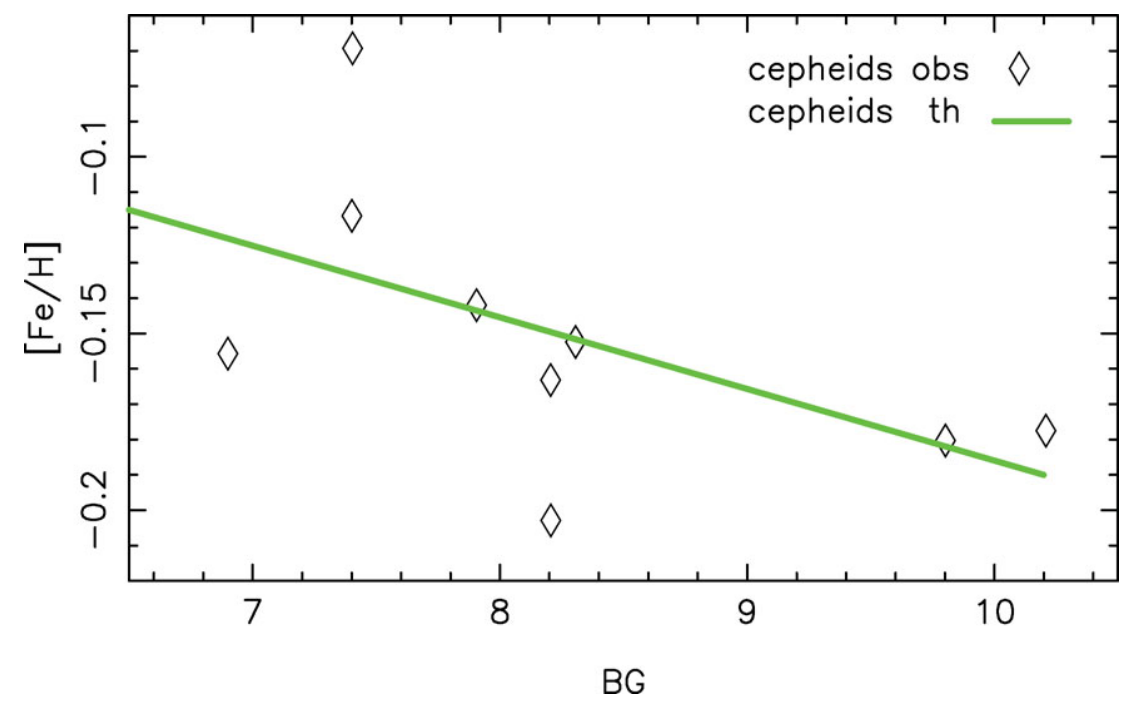

Figure 1. Galactocentric abundances using Cepheids as pulsators.

\section{References}

Cox, A. 1980, ARAESA, 18, 15

Suran, M. D. 1985, Top. Astrophys. Astron. Space Sci., 1, 1

Suran, M. D. 1986, Top. Astrophys. Astron. Space Sci., 2, 28

Suran, M. D. 1990, Contrib. Astron. Obs. Skalnat Pleso, 20, 77 